\title{
La Carta de Derechos Fundamentales de la Unión Europea desde la perspectiva de un jurista español
}

Miguel Revenga Sánchez

La Carta de Derechos Fundamentales de la Unión Europea («La Carta», en lo sucesivo), proclamada por el Consejo Europeo en diciembre de 2000, es uno de esos textos que hacen las delicias de cualquier jurista, teórico o práctico del Derecho. Es un texto que entusiasma por su sencillez, cincuenticuatro artículos bien ordenados y sistematizados en capítulos, y un breve Preámbulo redactado con claridad diáfana. Se trata de un texto normativo bien diferente de los que estamos acostumbrados a leer en el ámbito del derecho Comunitario. Un texto que, hablando en líneas generales, «se entiende» sin necesidad de ser un experto, pero cuya densidad normativa y cuyas implicaciones simbólicas en lo político son enormes. No es de extrañar que en el año apenas transcurrido desde su aprobación hayan proliferado las Discusiones y los Convenios dedicados a analizarla, fruto de los cuales contamos ya con abundantes papers y alguna monografia . Porque además, en torno a la Carta, concurre una circunstancia que hace de ella un instrumento especialmente sugestivo para el diálogo científico. La Carta es, por el momento, puro law on the books, un texto precioso, pero falto en absoluto de práctica aplicativa que permita constatar lo acertado o desacertado de las diagnosis que se van realizando. Es como si, a resultas del plazo de espera marcado por el Consejo de Niza, se hubiera invitado a la comunidad científica a especular y a arrojar luces, antes de tomar cualquier decisión comprometida sobre el destino final del Documento.

La particular contribución que deseo realizar a ese debate es una enmarcada en límites bien precisos: en sentido negativo, los que resultan de mi condición de jurista teórico, constitucionalista por más señas, y más familiarizado, por tanto, con las "grandes cuestiones" de la 
constitucionalización de Europa, que con los problemas de detalle derivados de la aplicación casuística del derecho comunitario. Y en sentido positivo, los que me impongo conscientemente, dadas mi procedencia y las características de la audiencia a la que tengo el honor de dirigirme. Hablo, pues, como el que soy : alguien interesado en cuestiones de teoría constitucional, que sigue, también como ciudadano, las discusiones sobre el futuro de Europa, y que enseña (y vive bajo el influjo de) la Constitución que los españoles nos dimos en 1978.

Me referiré brevemente, en primer lugar, al contexto político y constitucional en el que se inserta la nueva Carta de derechos (I). Analizaré luego, siempre desde la perspectiva española, la sistemática utilizada por la misma para recoger los derechos (II), y contemplaré, por fin, las incógnitas que la Carta abre de cara a una posible "constitucionalización" de Europa (III).

\section{La Carta de derechos en el contexto constitucional de la integración}

En la década transcurrida entre 1975 y 1985 (una década prodigiosa para muchos) España pasó de ser una dictadura pintoresca en el contexto europeo a estar integrada, como democracia plenamente normalizada, en la Comunidad Europea. La mayor parte de las fuerzas de oposición al franquismo venía considerando la integración en Europa, desde los años sesenta, como el destino deseable para la España democrática. Por eso no es de extrañar que, al tiempo de elaborarse la Constitución, se tuviera bien presente ese objetivo político y se instaurara un cauce expreso para hacerlo compatible con la norma constitucional. Dicho cauce expreso quedó recogido en el Capítulo que la Constitución consagra a los Tratados Internacionales y precisamente en el artículo que lo abre : «mediante ley orgánica - dice el artículo $93^{\circ}$ - se podrá autorizar la celebración de Tratados por los que se atribuya a una organización o institución internacional el ejercicio de competencias derivadas de la Constitución». El artículo habla, como se ve, de atribuir el ejercicio de competencias, no de ceder la competencia misma. Con tan sutil juego lingüístico se pretendió quizá calmar los ánimos de los "soberanistas" a ultranza, sentando una idea no del todo desacertada a la luz de los avatares jurídicos sufridos por los Tratados en los últimos años : la de que el fundamento último de la integración reside en la soberanía particulari- 
zada de cada Estado-miembro y, relacionada con ella, al menos en el plano teórico, la de que la cesión del ejercicio de las competencias podría estar sujeta a revocación.

Bien sabían quienes hicieron la Constitución que esa posibilidad pertenecía más a la política ficción que a la realidad de unas Comunidades integradas entonces por nueve Estados (los seis originarios más el Reino Unido, Irlanda y Dinamarca), pero no deja de ser curioso comparar el lenguaje calculadamente ambiguo del artículo $93^{\circ}$, con la rotundidad del utilizado para repartir competencias entre el Estado y las Comunidades Autónomas. Tanto conocían las implicaciones jurídicopolíticas de una eventual integración, que para reasegurar que ella no fuera a producir un conflicto irresoluble con la norma constitucional, se contempló la posibilidad de un control preventivo sobre Tratados que pudieran incluir estipulaciones contrarias a ella, a cargo del Tribunal Constitucional. Así viene establecido en el artículo $95^{\circ} \mathrm{CE}$, que obliga a poner en marcha la operación de reforma constitucional - aunque el artículo habla de "revisión" - con carácter previo a la celebración del Tratado, cuando se apreciara incompatibilidad entre el texto de éste y la Constitución. Tras la supresión, en 1985, del Recurso previo de inconstitucionalidad contra proyectos de leyes orgánicas, que recogía la Ley Orgánica del Tribunal, ese cauce del artículo $95^{\circ} \mathrm{CE}$ ha quedado como la única atribución de carácter preventivo, al estilo francés, dentro de un sistema de jurisdicción constitucional que, como se sabe, incluye también elementos característicos de los sistemas italiano (cuestión de inconstitucionalidad) y alemán (recurso de amparo).

Con tan escasos fundamentos constitucionales, objeto de abundante discusión doctrinal sobre el alcance del poder de integración que confieren, lo cierto es que la Ley Orgánica $\mathrm{N}^{\circ} 10 / 1985$, de 2 de agosto, autorizó la adhesión de España a las Comunidades (con efectos a $1^{\circ}$ de enero de 1986 para la incorporación del derecho comunitario), y otro tanto hicieron varias leyes orgánicas para autorizar la ratificación del Acta Única Europea (LO 4/1986), el Tratado de la Unión Europea (LO 10/1992) y el Tratado de Ámsterdam (LO 9/1998). De todas esas ocasiones, la más conflictiva fue la que dio lugar, en 1992, a la reforma de Maastricht. Ella fue la que ha dado lugar a la única "Declaración", por el momento, del Tribunal Constitucional a través del cauce del artículo $95^{\circ} \mathrm{CE}$. Y ella ha sido también la que originó la única reforma experimentada por la Constitución española en sus veintitrés años de vida. La reforma, de bien 
limitado alcance, afectó al artículo $13^{\circ}$, para propiciar que los nacionales de países comunitarios pudieran concurrir como candidatos a las elecciones locales.

Hoy es un lugar común afirmar que el «manto de silencio constitucional" que cubre en España todo lo relativo al proceso de construcción europea, es algo insostenible. A raíz de Maastricht, el proceso de reforma, realizado sin intervención directa del electorado por la vía del referéndum, y culminado en pleno período vacacional, en agosto de 1992, no captó ni mucho menos el interés de la opinión pública. Quizá porque de todo lo decidido en Maastricht, la posibilidad del sufragio pasivo, como un aspecto de la incipiente ciudadanía comunitaria, no es precisamente lo más importante. El caso es que pesó el argumento de la "economía de la reforma» (no tocar la Constitución más de lo estrictamente imprescindible), y luego, en los años transcurridos desde entonces, es como si hubiéramos caído en una especie de fetichismo constitucional que tiende a ver en las propuestas de reforma no sé qué peligros desestabilizadores.

Incidentalmente cabe decir que, a través del artículo $93^{\circ}$, hemos tenido también, no hace mucho, otra cesión de competencias a instancias supranacionales, esta vez con motivo de la ratificación por España del Tratado sobre el Tribunal Penal Internacional (LO 6/2000).

Pero el proceso de integración no está sólo regido por las disposiciones que acabamos de recordar. A veces se olvida que, fuera del Capítulo dedicado a los Tratados Internacionales, y abriendo precisamente el Título que la Constitución consagra a los derechos fundamentales, encontramos un artículo que atañe de lleno a nuestro tema. Me refiero al artículo $10^{\circ}$, con sus dos Apartados, a cual más importante. En el primero se recoge lo que hoy solemos llamar la vertiente objetiva de los derechos, esto es, su condición de «fundamento del orden político y de la paz social». Y en el segundo se incluye la única disposición-guía en materia de interpretación de los derechos - quizá sería mejor decir del Derecho a secas - que podemos encontrar en la Constitución :

"Las normas relativas a los derechos fundamentales y a las libertades que la Constitución reconoce se interpretarán de conformidad con la Declaración Universal de Derechos Humanos y los tratados y acuerdos internacionales sobre las mismas materias ratificados por España”. 
En un principio se tendió a decir que esta disposición no añadía nada al artículo $96^{\circ} \mathrm{CE}$, aquel que establece la recepción en el ordenamiento interno español de los Tratados válidamente celebrados. Pero la evolución de las cosas, y especialmente el abundante uso que el Tribunal Constitucional hizo enseguida de tal cláusula para explicar sus construcciones en materia de interpretación de los derechos, permitió que empezáramos a ser conscientes de su significado. Hoy nadie pone en duda el lugar central, y no meramente subsidiario, o a los solos efectos de interpretación sistemática, que el artículo 10.2 CE viene desempeñando en la elaboración dogmática de nuestro sistema de los derechos fundamentales. Sobre la estela del camino abierto por el artículo $16^{\circ}$ de la Constitución portuguesa de 1976, en el que se inspiró el artículo 10.2 de la nuestra, la irrupción de lo que Häberle llama «el quinto método de interpretación", yuxtapuesto a los cuatro tradicionales de Savigny, es una de las marcas distintivas del constitucionalismo más reciente.

El método comparado, la apertura dialogante hacia el exterior, de cara a la interpretación de los derechos, están haciendo emerger, cada vez con más fuerza, un Derecho Constitucional Común de los derechos. Una cultura de los derechos (que se corresponde con una concepción del Derecho Constitucional como ciencia cultural) que cada vez es menos dependiente del marco estatal y que se va afirmando y reafirmando ante nuestros ojos a lo largo y ancho de la "gran Europa", la del Consejo y la del Tribunal Europeo de Derechos Humanos.

Tengo para mí que esa capacidad de integración de los derechos fundamentales se va a ver potenciada, de manera espectacular, por la Carta. Y creo también que un efecto de la Carta, que ya se deja sentir, es el de superar para siempre las relaciones de contraste y conflicto que los derechos reconocidos por cada Constitución nacional han tendido a manifestar con respecto al Derecho comunitario.

\section{La Carta y su sistemática}

Para cualquiera que esté acostumbrado a leer textos jurídicos en materia de derechos, lo primero que le llama la atención de la Carta europea es su distribución sistemática. Los derechos se agrupan en función de unos valores o unos principios fuertes (dignidad, libertad, igualdad, solidaridad, ciudadanía y justicia), que la Unión, según se afirma en el Preám- 
bulo, reconoce. Se reconocen derechos, libertades y principios, y estos últimos por sí mismos, sin perjuicio de que, en algún caso, se reformulen además como derechos. Acerca de esa positivización de los principios y de los valores, y en torno a la ausencia de cualquier gradación o jerarquía entre los derechos y principios que la Carta recoge, es sobre lo que me gustaría realizar alguna consideración desde la perspectiva española.

Los principios comunes en materia de derechos fueron, como es sabido, el expediente del que se valió el Tribunal de Justicia para evitar un choque frontal entre el Derecho nacional y el Derecho comunitario. El Tratado de la Unión reforzó y multiplicó en 1992 la referencia a los principios y a las tradiciones constitucionales comunes, que han pasado a ser desde entonces - y así lo atestigua el Preámbulo de la Carta - el nexo de unión más consistente. Lo que llama la atención no es el valor simbólico, en lo político, de esa proclamación de fe en los valores comunes, sino el auge, en lo jurídico, de una técnica declarativa y una práctica aplicativa que es cada vez más "principialista" $y$, en consecuencia, menos tributaria de categorías y formulaciones concebidas en circunstancias históricas distintas, por no decir contrapuestas, a las del constitucionalismo democrático de nuestros días.

La experiencia española nos parece, desde este punto de vista, bastante interesante. Como es sabido, la CE de 1978 es pródiga en el reconocimiento de valores y principios. El artículo $1^{\circ}$ propugna unos valores superiores del ordenamiento jurídico (libertad, justicia, igualdad y pluralismo político), y hace de la defensa de ellos el objetivo último del Estado social y democrático de derecho que se constituye. El 10.1 eleva la dignidad de la persona, con los derechos inviolables que le son inherentes, a la posición de fundamento del orden político y de la paz social. Y luego el considerable grado de abstracción de esos valores se concreta en principios (el caso paradigmático, pero no el único, es del artículo 9.3) y/o en derechos (como ocurre, por ejemplo, con la igualdad en el artículo $\left.14^{\circ}\right)$.

El deseo de dotar a la Constitución de fuerza normativa, algo inédito en nuestra historia, explica el poco entusiasmo, por no decir escepticismo combativo, con que los valores y principios fueron recibidos en sede doctrinal. Sobre esa actitud pesaba, sin duda, el recuerdo de una utilización abiertamente conservadora de los viejos "principios generales del derecho", del Código Civil. Pero también subyacía algo que, aquí en Italia, conocieron muy bien durante los años iniciales de andadura cons- 
titucional : la separación entre un Derecho programático y vacío de consecuencias prácticas (no por casualidad, el de la Constitución y sobre todo el de su parte llamada tradicionalmente «dogmática»), y el Derecho verdadero, el que comienza más allá de la Constitución, en la medida en que así lo quieran los encargados de desarrollarla.

Nuestro TC combatió, desde sus primeras Sentencias, un punto de vista como el apuntado. Pero no por ello se decidió a embarcarse en una jurisprudencia de valores. Y ello pese a que muy pronto - desde la STC $11 / 1981$ - un concepto como el del "contenido esencial" de los derechos, verdadero «límite de los límites» de éstos, según el artículo 53.1 $\mathrm{CE}$, dio lugar a muy curiosas disquisiciones por parte de algún magistrado para aproximarse al significado de aquel concepto. Hasta es posible que la pujanza con que se recurrió al método comparado pudiera ser expresión de las cautelas del Tribunal a la hora de recurrir a los valores y a los principios. En todo caso es interesante comprobar cómo el miedo al recurso de los valores ha ido declinando en la misma medida en que las líneas jurisprudenciales se han ido haciendo más sólidas y se han ido perfilando mejor el uso de categorías - clave en la justicia constitucional de nuestros días, como la razonabilidad o la proporcionalidad.

Un indicio de ese cambio de actitud con respecto al recurso a valores podemos encontrarlo comparando el voto particular que realizara el magistrado Tomás y Valiente a la STC 53/1985, sobre la Ley despenalizadora del aborto, y la actitud reflejada por dicho magistrado, años más tarde, en un trabajo publicado poco antes de su vil asesinato a manos de la banda terrorista ETA. En el primer caso, el del voto particular, vemos un posicionamiento contrario a cualquier contaminación de la "filosofía de los valores" sobre la tarea de la jurisdicción constitucional; en el segundo, asistimos a una reivindicación entusiasta de la fuerza normativa de los valores (que no son, en las propias palabras de ' Comás y Valiente, "música celestial") y a una defensa de la "moralidad política", a la Dworkin, en el campo de la interpretación del Derecho.

Bien sabemos que una cosa es la ética de la responsabilidad, en la labor de magistrado, y otra la ética de la convicción en la de profesor y teórico. Pero ese cambio de perspectiva en un jurista de tanto influjo como el malogrado ex Presidente del Tribunal simboliza, creo, la evolución de una concepción del Derecho hoy bien abierta a valores. Aunque sólo sea de pasada, tampoco sería justo dejar de mencionar que probablemente no hay libro en la doctrina española que haya contribuido 
tanto a ese cambio de perspectiva como el Diritto mite, de Gustavo Zagrebelsky.

Por lo que se refiere a la gradación y a la jerarquía (o más bien ausencia de ella) en los derechos reconocidos en la Carta, también eso es llamativo para un jurista español. De nuevo la obsesión de nuestro constituyente por hacer del texto constitucional algo operativo en materia de derechos, unido al deseo de proclamar cuantos más derechos mejor, llevó a que el Título a ellos consagrado esconda una "orografía" de los derechos que los distingue y jerarquiza, ya desde el nomen iuris y abre una brecha insalvable entre unos genuinos derechos fundamentales, $\mathrm{y}$ unos heterogéneos e imprecisos derechos sociales, agrupados bajo la incoherente rúbrica - ahora con intención de menoscabar su valor - de principios rectores de la política social y económica. Para empeorar las cosas, entre unos y otros todavía hay una imprecisa categoría de derechos constitucionales (por ejemplo el derecho de propiedad, el de contraer matrimonio o el derecho a la negociación colectiva laboral), que no se sabe muy bien dónde emplazar, pues no tienen recurso de amparo - verdadera marca distintiva del nivel máximo de garantías - pero superan con creces el valor meramente informador de la legislación positiva y la práctica de los poderes públicos, que es lo que corresponde, según el artículo 53.3 CE, a esos llamados principios rectores.

El concepto de derecho fundamental es todavía, entre nosotros, a la luz de la Constitución, algo bastante conflictivo y sobre lo que seguimos discutiendo. Por eso choca la horizontalidad al respecto de la Carta. No hay en ella nada que permita hablar de derechos de primer y de segundo o tercer nivel, aunque ello no significa que la vieja contraposición entre los derechos de libertad y los derechos de prestación - o, si se prefiere, desde una perspectiva histórica, los que separan la primera y segunda de la tercera generación de los derechos llamados comúnmente sociales - no siga ejerciendo un influjo, quizá decisivo, sobre el lenguaje de la Carta. Por ahí han asomado ya las primeras críticas y los primeros asombros, como si cupiera esperar más de ella, sobre todo considerando el paso adelante que supuso la adhesión de la Unión a la Carta Social Europea, en los términos que hoy figuran en los artículos $136^{\circ}$ y $137^{\circ}$ del Tratado de la Comunidad Europea, tras la reforma de Ámsterdam. Muchos de los derechos agrupados bajo el epígrafe "Solidaridad" aparecen, en efecto, sujetos a un reenvío condicionante al derecho comunitario y a las "legislaciones y prácticas nacionales». Sobre todo, llama la 
atención esta última referencia a las prácticas nacionales, una técnica ciertamente insólita para delimitar el alcance de un derecho, que parece pensada para consolidar lo existente, neutralizando cualquier dimensión transformadora de una realidad y unos condicionantes que se asumen de la manera más descarnada y acrítica.

Otras diferenciaciones deducibles de la Carta son las que separan a la mayor parte de los derechos, tenidos por tradicionales, del pequeño, pero significativo número de derechos y prohibiciones, de carácter nuevo. Dada la timidez de nuestro TC a la hora de «descubrir» derechos no escritos en el texto constitucional, la Carta puede actuar, al respecto, como un buen incentivo. Para el debate actualmente en curso en España sobre la equiparación del matrimonio heterosexual a las Uniones de hecho, homo o heterosexuales, la Carta no parece, sin embargo, demasiado innovadora, pues, pese a la prohibición de discriminación por razón de la tendencia sexual (artículo $21^{\circ}$ ), el derecho a casarse y a fundar una familia (artículo $9^{\circ}$ ) aparece también sujeto a la cláusula de remisión a las legislaciones nacionales.

En cambio las diferenciaciones de la Carta atinentes al título en virtud del cual se reconocen los derechos, qua persona o qua miembro activo de una determinada comunidad política, es decir, los distingos y jerarquizaciones relativas al estatuto de ciudadanía, sí podrían influir sobre el conflicto que enfrenta en España a los defensores y a los detractores de la actual Ley de Extranjería. En los próximos meses, el TC habrá de dilucidar si se ajusta o no a la Constitución sujetar el ejercicio de los derechos de reunión y asociación, por parte de los extranjeros, al requisito de haber obtenido autorización de estancia o residencia en España (arts. $7^{\circ}$ y $8^{\circ}$ de la LO 4/2000). Por haber mantenido que se trata de un límite desproporcionado y, en consecuencia, inconstitucional, hemos comprobado con satisfacción que la Carta reconoce ambos (lerechos, para la defensa de sus intereses, a «todas las personas». Aún cuando el ámbito de aplicación de la Carta está circunscrito, en el caso de los Estados, a la aplicación del Derecho de la Unión (artículo 51.1), no tenemos duda alguna acerca del influjo que la Carta va a ejercer - y, de hecho, está ejerciendo ya - a nivel interno y supranacional, en la interpretación de cualquier sistema de los derechos fundamentales. 


\section{La Carta y la constitucionalización de Europa}

La proximidad del semestre que se inicia el $1^{\circ}$ de enero, en el que corresponderá a España la presidencia de la Unión Europea, ha hecho que los líderes de los dos grandes partidos españoles (el Partido Popular, actualmente en el Gobierno y el Partido Socialista) hayan acudido no hace mucho a Bruselas a exponer las líneas maestras de su proyecto de Europa. Aunque las ideas de una y otra fuerza política difieren en cuestiones de detalle, las dos se sitúan en una especie de punto equidistante entre la Europa federal, preconizada por el canciller alemán, y la Europa de las naciones confederadas, auspiciada por el primer ministro francés. Ambas visiones coinciden en reivindicar "más Europa", algo que los políticos españoles ven especialmente atractivo en el campo de las políticas de seguridad y defensa común, como un medio (en trance de lograrse tras el 11 de septiembre) de comprometer a la Unión en la lucha contra el terrorismo etarra.

Curiosamente, el presidente Aznar y el líder del Partido Socialista, Rodríguez Zapatero, han hablado también de la necesidad de constitucionalizar los Tratados. Sea cual fuere el significado de tal objetivo, lo que parece evidente es que «constitucionalizar los Tratados» quiere decir, en ese contexto, hacer de ellos algo más simple e inteligible para el ciudadano medio. La actual dispersión de las normas básicas europeas en sucesivas versiones de cuatro grandes Tratados, y un número de Protocolos y Declaraciones que supera holgadamente la treintena, no contribuye en nada a la claridad : ¡más de 800 artículos, detallistas a veces hasta lo mareante, para no resolver los interrogantes básicos que están dentro de cualquier Constitución! Aunque sólo fuera por el logro simplificador plasmado en la Carta, los esfuerzos de quienes la han elaborado, alumbrados por el modesto propósito de dotar de "visibilidad" a un incipiente sistema europeo de los derechos, habría valido la pena.

Desde una visión optimista de las cosas, la Carta puede presentarse como un paso decisivo en el camino de la constitucionalización de Europa. De ella se ha dicho que viene a culminar, por ahora, un decurso de la historia de la construcción europea, que se ha ido mostrando sucesivamente cada vez más ambicioso en materia de reconocimiento y garantía de los derechos. A partir del originario deseo de paz y bienestar, presente en los momentos iniciales, cada una de las grandes etapas de ese recorrido ha ido acompañada de actitudes combativas en defensa de los 
derechos. Si en la década de 1970, y hasta la primera ampliación, el protagonismo fue del Tribunal de Justicia, en la década de 1980, coincidiendo con el ingreso de Grecia, Portugal y España, fue el Parlamento (y en menor medida la Comisión) quien más se destacó en reclamar una incorporación sistemática de los derechos.

Tras los cambios decisivos que hemos vivido en la década de 1990, quizá ha llegado el momento de que la razón jurídica, y las razones técnica, democrática y politico-estatal (es decir, las que pueden encarnar, respectivamente, el Tribunal de Justicia, la Comisión, el Parlamento y el Consejo), sincronicen su tiempo histórico para adaptarlo a la hora constitucional. La Carta representaría entonces un punto de llegada y también un punto de partida culminado de acuerdo con las exigencias de la más rigurosa ortodoxia constitucional : proclamando ideales y definiendo las posiciones básicas de los individuos antes de empezar a discutir todo lo demás. En su haber efectivo hay que incluir el haber dotado a Europa de una lingua franca, la de los derechos fundamentales, y haber insuflado anima a un ideal, oscurecido por el día a día de una "eurocracia" fría y alejada de los ciudadanos. Con su elaboración, se ha realizado además un ensayo de laboratorio constitucional y una práctica de democracia deliberativa, hasta ahora inéditos en el contexto europeo. El éxito de la empresa, en un plazo de tiempo decididamente corto, demuestra que nada es imposible cuando concurre imaginación y coraje político.

La Carta es hoy por hoy - según las palabras que utilizara Bagehot para describir la Constitución inglesa de su tiempo- dignified part de una inexistente Constitución europea. Pero la cualidad esencial de ese fragmento consiste en que también es ya efficient part de un proyecto político, que se nos muestra como una de las pocas utopías que tenemos a nuestro alcance. 